\title{
Knockdown of CREB1 inhibits tumor growth of human gastric cancer in vitro and in vivo
}

\author{
MIN RAO ${ }^{1}$, YONGGANG ZHU ${ }^{3}$, XIAOXIA CONG ${ }^{2}$ and QIANXUN $\mathrm{LI}^{2}$ \\ ${ }^{1}$ Department of Gastroenterology, The First Hospital of Jilin University; ${ }^{2}$ Department of Gastroenterology, \\ The People's Hospital of Jilin Province, Changchun, Jilin 130021; ${ }^{3}$ Department of Radiotherapy, \\ China-Japan Union Hospital of Jilin University, Changchun, Jilin 130033, P.R. China
}

Received September 21, 2016; Accepted February 24, 2017

DOI: $10.3892 /$ or.2017.5636

\begin{abstract}
AMP responsive element binding protein 1 (CREB1) gene, has been reported to play crucial roles in tumor progression and development in various types of cancer. Little is known, however, about its role and underlying mechanism in gastric cancer (GC). Herein, we investigated the biological roles and molecular mechanism of CREB1 in GC. The expression level was determined in four GC cell lines by quantitative RT-PCR and western blotting. Recombinant expression vector carrying small interfering RNA (siRNA) targeting CREB1 was constructed and then transfected into human GC cell line (SGC-7901). Cell proliferation, colony formation, cycle distribution, migration and invasion in vitro were determined by MTT, colony forming, flow cytometry, would healing and Transwell invasion assays after CREB1 knockdown. Tumor growth in vivo was assessed by measurement of tumor volume and weight in a nude mouse model. We found that CREB1 was highly expressed in the human GC cell lines. We also showed that knockdown of CREB1 in SGC-7901 cells significantly inhibited cell proliferation, colony formation, migration and invasion and induced cell arrest at G1/G0 phase in vitro, as well as suppressed tumor growth in viyo. In addition, CREB1 knockdown was able to significantly reduce expression of its downstream target genes cyclin D1, Bcl-2 and MMP-9 in vitro and in vivo. These findings suggest that CREB1 may be a potential therapeutic target for the treatment of gastric cancer.
\end{abstract}

\section{Introduction}

Worldwide, gastric cancer (GC) is the is the fourth most malignant cancer and the second most frequent cause of cancer-related mortalities (1). Despite improvements in surgical techniques and multimodal therapy, the prognosis for GC patients

Correspondence to: Dr Qianxun Li, Department of Gastroenterology, The People's Hospital of Jilin Province, Changchun, Jilin 130021, P.R. China

E-mail: liqianxun1452@126.com

Key words: gastric cancer, cAMP responsive element binding protein 1 , tumor growth, proliferation remains poor mainly due to tumor cell unlimited proliferation and strong invasive and metastasis ability (2). Conventional GC is characterized by genetic and epigenetic alterations that influence key cellular pathways involved in growth and metastasis of GC (3). Therefore, improved understanding of the molecular mechanisms involved in GC initiation and development would likely contribute to find therapeutic target for GC therapy, thereby further improving the overall outcome for patients with GC.

CAMP response element-binding protein (CREB1) is a nuclear transcription factor that belongs to the basic leucine zipper (bZIP) family (4). It has been shown that activated CREB1 could bind to the conserved cAMP-responsive element (CRE) on the promoter and mediates transcriptional responses to a variety of stimuli, such as neurotransmitters, hormones, membrane depolarization, and growth and neurotrophic factors $(5,6)$. It has been reported to serve as a mediator between different signal pathways and the transcription of downstream target genes, such as the cell apoptosis-related genes Bcl-2, the cell invasion-related gene MMP9, the cell cycle-related genes cyclinA1, cyclinB1, and cyclin D1 (7). It has been found to be upregulated in a variety of cancers including non-small cell lung carcinoma (8), breast cancer (9), acute myeloid leukemia (10), colorectal cancer (11), and gliomas (12), suggesting CREB1 as an oncogene in these types of cancer. In addition, CREB1 could regulate proliferation, invasion, and metastasis of tumor cells (13-15). Previously a study showed that CREB1 was highly expressed and correlated with lymph node metastasis, distant metastasis and tumor stage and poor outcome in gastric cancer (16). However, the biological roles of CREB1 remains unknown in GC. The aims of this study were to investigate the role of CREB1 and evaluate its mechanism under gastric carcinogenesis and metastasis.

\section{Materials and methods}

Cell lines and cell culture. The immortalized gastric epithelial cell GES-1 and four human GC cell lines (BGC-823, SGC-7901, MKN-45, AGS) were all obtained from the Cell Bank of Shanghai Institute of Cell Biology (Shanghai, China). The cells were maintained in Roswell Park Memorial Institute 1640 (RPMI-1640, HyClone, Logan, UT, USA) supplemented with $10 \%$ fetal bovine serum (HyClone) in an incubator at $37^{\circ} \mathrm{C}$. 
siRNA design and production of recombinant adenovirus. The siRNA target design tools (Ambion, Austin, TX, USA) were used to design CREB1 and negative control scramble shRNA sequences. The synthesized oligonucleotides containing specific target sequence, a loop, the reverse complement of the target sequence, a stop codon for U6 promoter and two sticky ends were synthesized by Takara (Dalian, China). siRNAs targeting CREB1 sequence (si-CREB1) and scramble control (si-Ctrl) sequence were as followed: si-CREB1 (sense) ACG GTGCCAACTCCAATTTAC; si-Ctrl: AATTCTCCGAACG TGTCACGT (sense). The si-CREB1 and si-Ctrl oligonucleotides were cloned into expressing plasmid pCDNA3.0-CMV (Invitrogen, Carlsbad, CA, USA), named as psi-CREB1 and psi-Ctrl. psi-CREB1 and psi-Ctrl plasmid and transfected into SGC-7901 cells using Oligofectamine ${ }^{\mathrm{TM}}$ Transfection (Invitrogen) according to the manufacturer's instructions. At $48 \mathrm{~h}$ post-transfection, the cells were harvested and knockdown efficiency was determined by quantitative RT-PCR (qRT-PCR) and western blot analysis.

Real-time quantitative RT-PCR ( $q R T$-PCR). Total RNA was isolated from cultured cells using TRIzol (Invitrogen) according to the manufacturer's instructions. RNA was reverse-transcribed into cDNA using Primescript ${ }^{\mathrm{TM}}$ RT reagent kit according to manufacturer's protocols (Takara). Quantitative real-time polymerase chain reaction (qPCR) assays were performed with SYBR Green Real-time PCR Master Mix (Toyobo, Osaka, Japan) under ABI 7900 Fast system (Applied Biosystems, Foster City, CA, USA). The primes of CREB1 and GAPDH (as an internal control) were as previously described (17). The PCR conditions were as follows: a pre-denaturing at $95^{\circ} \mathrm{C}$ for $3 \mathrm{~min}$, followed by 40 cycles of denaturation at $95^{\circ} \mathrm{C}$ for $10 \mathrm{sec}$, annealing/extension at $60^{\circ} \mathrm{C}$ for $30 \mathrm{sec}$, final extention $72^{\circ} \mathrm{C}$ for $5 \mathrm{~min}$. The amplification specificity was checked by melting curve analysis. Relative CREB1 mRNA expression were determined by Bioneer Exicycler $^{\mathrm{TM}}$ analysis software (Bioneer Corp., Daejeon, Korea) using the $2^{-\Delta \Delta C T}$ method.

Western blotting. The cultured cells or tumor tissues were harvested and lysed in lysis buffer $(50 \mathrm{Mm}$ Tris- $\mathrm{HCl}$ (pH 7.5), $20 \mathrm{mM} \mathrm{NaCl}, 5 \mathrm{mM}$ EDTA, $1 \%$ TX-100, 0.1\% SDS, $5 \%$ glycerol + protease inhibitors) on ice for $30 \mathrm{~min}$, and then centrifuged at $20,000 \mathrm{x} \mathrm{g}$ at $4^{\circ} \mathrm{C}$ for $15 \mathrm{~min}$. The supernatant was collected and total protein concentration was determined using the Pierce bicinchoninic acid Protein Assay kit (Thermo Fisher Scientific, Waltham, MA, USA) with bovine serum albumin (BSA) as a standard control. Equal amounts of protein $(30 \mu \mathrm{g})$ were separated by $8-10 \%$ SDS-polyacrylamide gels (SDS-PAGE) and transferred to polyvinylidene difluoride (PVDF) membranes (Millipore, Bedford, MA, USA). After blocking for $1 \mathrm{~h}$ with 5\% skimmed milk in TBS buffer $(10 \mathrm{mM}$ Tris, $150 \mathrm{mM} \mathrm{NaCl})$, the membrane was immunoblotted with specific primary antibodies for $1 \mathrm{~h}$ at room temperature, and then incubated with corresponding horseradish peroxidase-conjugated secondary antibody for $2 \mathrm{~h}$ at room temperature. Protein bands were observed with enhanced chemiluminescence reagent (ECL, Amersham, GE Healthcare, Velizy-Villacoublay, France) and exposed to $\mathrm{X}$-ray film. The primary antibodies used in the western blots were as follows: mouse anti-human CREB1 (1:500 dilution, Santa Cruz Biotechnology, Santa Cruz, CA, USA), mouse anti-human Bcl-2 (1:1000 dilution, Santa Cruz Biotechnology), mouse anti-human cylin D1 (1:800 dilution, Santa Cruz Biotechnology), mouse anti-human MMP9 (1:1000 dilution, Santa Cruz Biotechnology), mouse anti-GAPDH (1:2000 dilution, Sigma-Aldrich, St. Louis, MO, USA). Secondary antibody used in this study was HRP-conjugated goat anti-mouse IgG (1:5000 dilution, Santa Cruz Biotechnology).

Cell proliferation and colony formation assays. To measure the effect of downregulation of CREB1 on cell proliferation, CCK-8 assay (Cell Counting Kit-8, Dojindo, Kumamoto, Japan) was performed. In brief, $5 \times 10^{3}$ of transfected cells were seeded into each well of a 96 -well plate and cultured for 24-96 h. At the end of different experimental periods $(24,48$, 72 and $96 \mathrm{~h}$ ), $10 \mu \mathrm{l}$ CCK-8 solution were added to each well followed by incubation for an additional $2 \mathrm{~h}$. When the media changed from red to yellow, the absorbance was measured at a wavelength of $450 \mathrm{~nm}$ using an enzyme-linked immunosorbent assay reader (Thermo Labsystems, Vantaa, Finland).

For the colony formation assay, SGC-7901 cells were incubated in 6-well plates for 18-24 h, and transfected with psi-CREB1 and psi-Ctrl plasmid for $48 \mathrm{~h}$. Transfected cells were resuspended and seeded onto 6-well plates at a density of 1000 cells/well and maintained in RPMI-1640 medium containing 10\% FBS. After 14 days of incubation, colonies were fixed with methanol and stained with $0.1 \%$ crystal violet for $15 \mathrm{~min}$, and then were photographed and counted under a light microscope (Olympus, Tokyo, Japan). The percentage colony formation was calculated by adjusting control to $100 \%$.

Cell cycle analysis. SGC-7901 cells transfected with psiCREB1 or psi-Ctrl were cultured in 6-well plates for $48 \mathrm{~h}$. Cells then were harvested by centrifugation at $2000 \mathrm{x}$ g for 5 min, washed twice with PBS, and then $75 \%$ ethanol at $4^{\circ} \mathrm{C}$ overnight. Then, cells were rehydrated and resuspended in PBS and incubated in $500-\mu 1$ staining solution $(10 \mu \mathrm{g} / \mathrm{ml}$ propidium iodide and $5 \mathrm{U} / \mathrm{ml} \mathrm{RNaseA}$ ) for $30 \mathrm{~min}$ at room temperature. Cell cycle distribution was determined using BD FACS Calibur Flow Cytometer (BD Biosciences, San Diego, CA, USA).

Cell migration and invasion assays. Cell migration was assessed using wound healing assays by measuring the movement of cells. In briefly, $1 \times 10^{5}$ transfected cells were seeded in 12-well plates in RPMI-1640 medium containing 10\% FBS. After $24 \mathrm{~h}$, a scratch was created through the confluent cell monolayer using a plastic micropipette tip. The wound closure was observed after $24 \mathrm{~h}$ and photographed under a microscope (Olympus).

Cell invasion assay was determined using BD BioCoat ${ }^{\mathrm{TM}}$ Matrigel invasion chambers (Becton Dickinson Labware, Bedford, MA, USA) according to the manufacturer's instructions. Briefly, $1 \times 10^{5}$ transfected cells in serum-free medium were plated in the upper of the membranes coated with Matrigel (BD Biosciences), while $600 \mu \mathrm{l}$ of medium containing $10 \%$ FBS with a chemoattractant was added to the lower chamber. After $48 \mathrm{~h}$, the non-invading cells were gently removed with a cotton swab, while the cells that had invaded to the lower side 

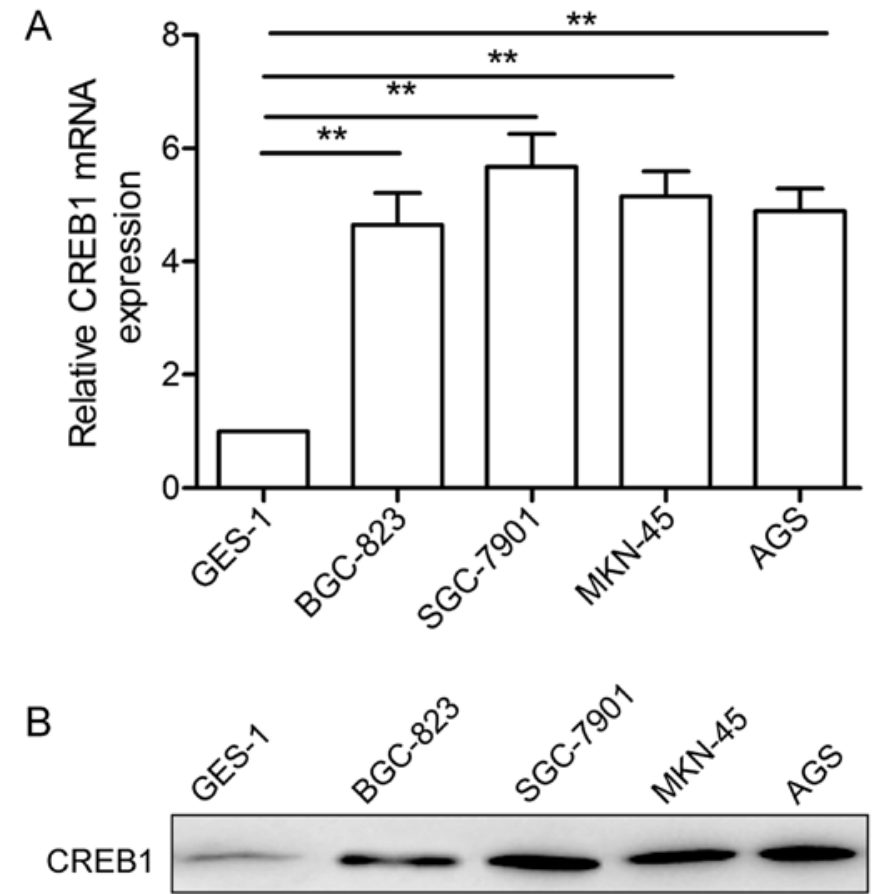

\section{GAPDH}

Figure 1. The expression of CREB1 is downregulated in human GC cell lines. (A) Relative CREB1 mRNA expression was determined in four human GC cell lines (BGC-823, SGC-7901, MKN-45, AGS) and immortalized gastric epithelial GES-1 cells by qRT-PCR. (B) The CREB1 protein expression was determined in four human GC cell lines (BGC-823, SGC-7901, MKN-45, AGS) and immortalized gastric epithelial GES-1 cells by western blotting. qRT-PCR and western blot assays were normalized to GAPDH. " $\mathrm{P}<0.01$

of the chamber were fixed in $20 \%$ methanol and stained with $0.1 \%$ crystal violet. The invasive cells were photographed, and quantified by counting them in random five fields by a light microscope (Olympus).

Tumor xenograft assay. Twenty 6-8-week-old male BALB mice were obtained from the Experimental Animal Center of Changchun Institute for Biological Sciences (Changchun, China), and were maintained under specific pathogenfree conditions and provided with normal food and water ad libitum. All animal experiments were approved by the Ethics Committees of the Disease Model Research Center of the People's Hospital of Jilin Province (Changchun, China).

The mice were fed with a normal pellet diet three days prior to the experimentation. SGC7901 cells in exponential growth phase were harvested and single-cell suspensions ( $2 \times 10^{6}$ cells in $100 \mu \mathrm{l}$ RPMI-1640 medium) were injected subcutaneously (s.c.) into the right side of the posterior flank of the mice. Tumor volume was monitored and calculated according to the formula: $\mathrm{V}=0.526 \times \mathrm{L}$ (length) $\mathrm{x} \mathrm{W}^{2}$ (width) by measuring tumor length and width every 3 days. When tumors grew to an average volume of $100 \mathrm{~mm}^{3}$, mice were randomly divided into psi-CREB1 and psi-Ctrl groups $(n=10$ in each group). The mice were inoculated with $50 \mu \mathrm{g} / 100 \mu \mathrm{l}$ per mouse via i.t. injection of psi-CREB1 or psi-Ctrl one time a week for four weeks, respectively. Tumor volume
A

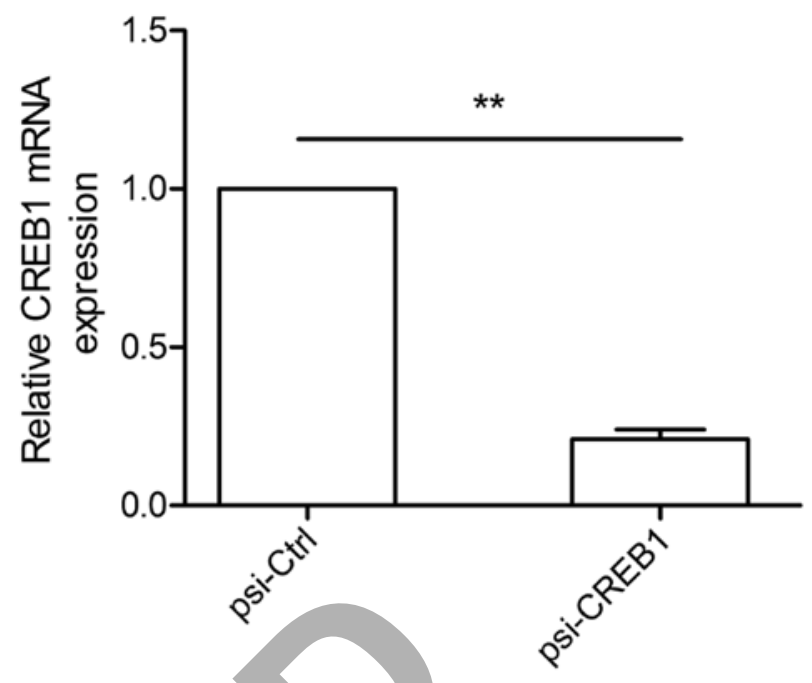

B psi-Ctrl psi-CREB1

CREB1 GAPDH

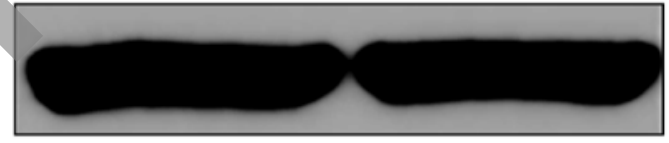

Figure 2. Downregulation of CREB1 expression by psi-CREB1 in human GC cells. (A) Relative CREB1 mRNA expression were determined in SGC7901 cells transfected with psi-CREB1 or psi-Ctrl plasmid by qRT-PCR. (B) CREB1 protein expression were determined in SGC-7901 cells transfected with psi-CREB1 or psi-Ctrl plasmid by western blotting. qRT-PCR and western blot assays were normalized to GAPDH. ${ }^{* *} \mathrm{P}<0.01$.

was monitored every 7 days. Mice were sacrificed one week after the final plasmid injection. Tumor tissues were excised and weighed. Some of the tissues were used for analysis of protein expression.

Statistical analysis. All data are presented as mean \pm standard deviation (SD) from at least three independent experiments with similar results. Statistical analyses were undertaken using the SPSS ${ }^{\circledR}$ statistical package, version 17.0 (SPSS Inc., Chicago, IL, USA). Statistical analysis between two samples was performed using Student's t-test. Statistical comparison of more than two groups was performed using one-way ANOVA. A $\mathrm{P}<0.05$ was considered to indicate a statistically significant difference.

\section{Results}

CREB1 is upregulated in gastric cancer cell lines. To detect CREB1 expression level in GC, we assessed its mRNA expression level and protein expression level in four human GC cell lines (BGC-823, SGC-7901, MKN-45, AGS) and immortalized gastric epithelial cell GES-1 by qRT-PCR and western blotting, respectively. Compared with gastric epithelial cells GES-1, CREB1 expression both at mRNA level (Fig. 1A) and protein level (Fig. 1B) was significantly upregulated in the four GC cell lines. SGC-7901 cell line 
A

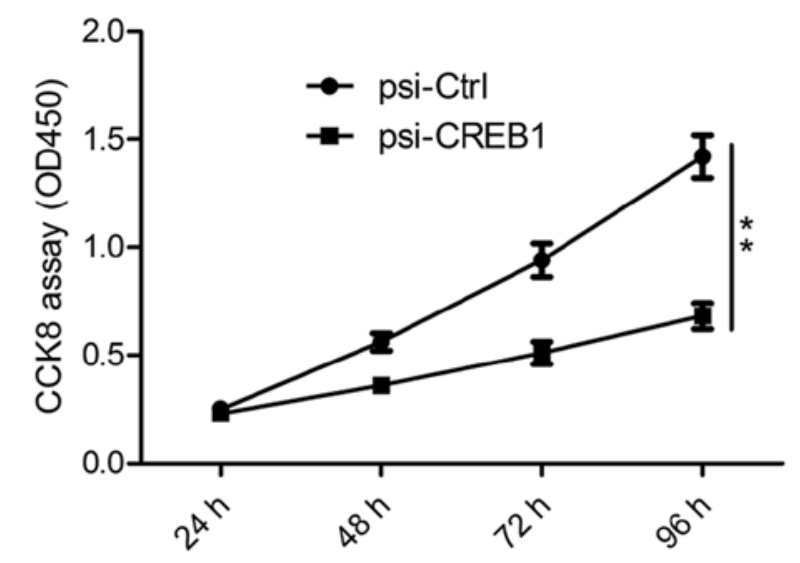

C

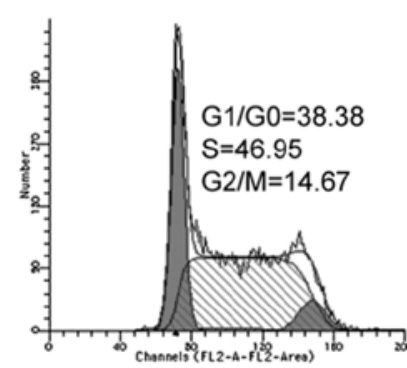

psi-Ctrl

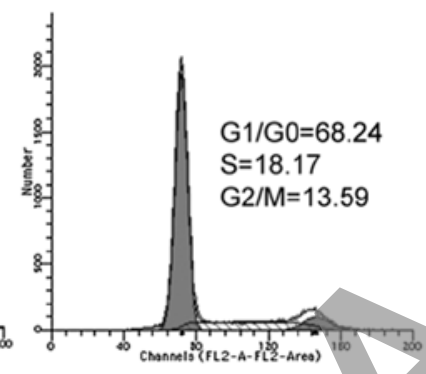

psi-CREB1

B

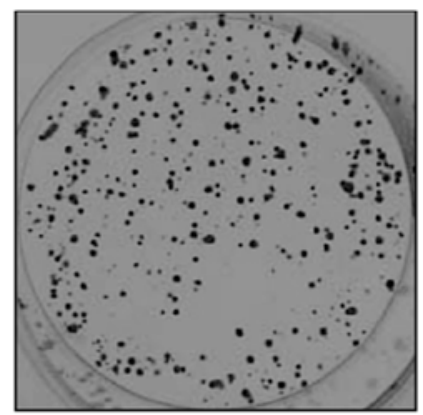

psi-Ctrl

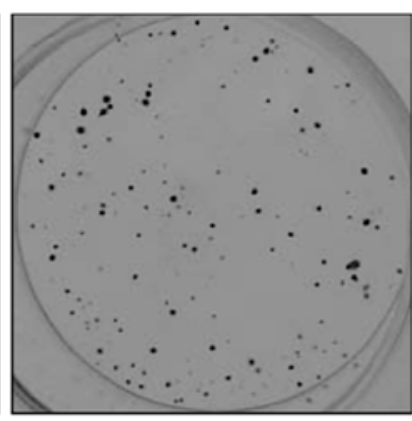

psi-CREB1

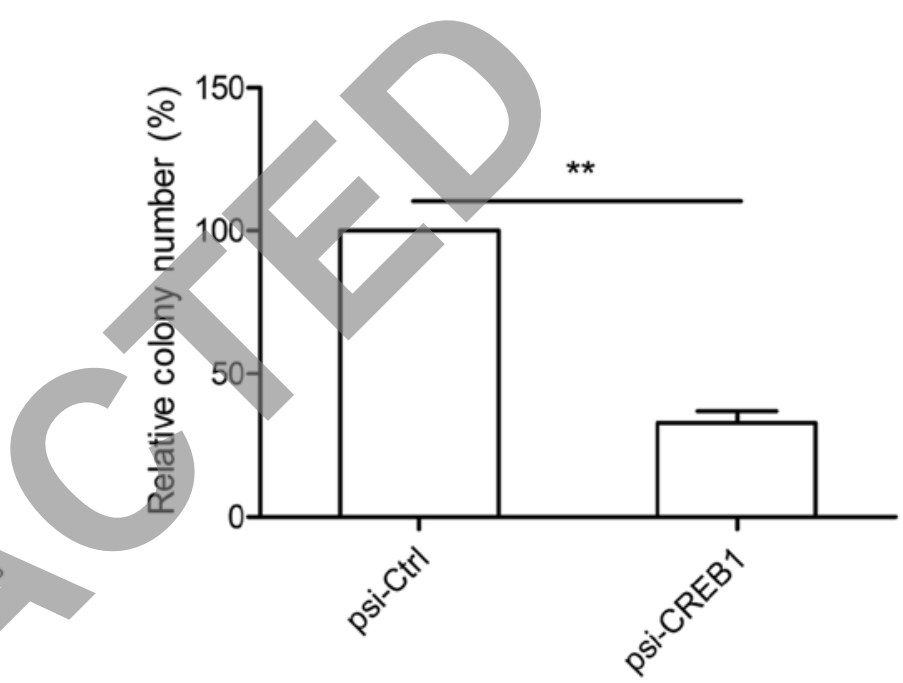

Figure 3. Downregulation of CREB1 inhibits proliferation and colony formation in GC cells. (A) Cell proliferation was determined in SGC-7901 cells transfected with psi-CREB1 or psi-Ctrl plasmid by CCK8 assay. (B) Cell colony formation was determined in SGC-7901 cells transfected with psi-CREB1 or psi-Ctrl plasmid by colony forming assay. (C) Cell cycle distribution was determined in SGC-7901 cells transfected with psi-CREB1 or psi-Ctrl plasmid by flow cytometry. ${ }^{* *} \mathrm{P}<0.01$

displayed a highest expression level of CREB1 among the cell lines (Fig. 1A and B), thus, it was selected as a model for further study.

Downregulation of CREBI expression by psi-CREBI in human GC cells. To study the biological role of CERB1 in $\mathrm{GC}$, we constructed pcDNA3-CMV vectors expressing small hairpin siRNA oligonucleotides targeting CREB1 (psi-CREB1) to selectively reduce CREB1 gene expression, then the pcDNA3-CMV vectors expressing CREB1 siRNA (psi-CREB1) or scramble siRNA (psi-Ctrl) were transfected into human SGC-7901 cells and cultured for $48 \mathrm{~h}$. Transfection efficiency was determined by measurement of mRNA and protein levels of CREB1 using qRT-PCR and western blotting, respectively. We found that knockout CREB1 by psi-CREB1 significantly decreased CREB1 expression at mRNA level (Fig. 2A) and protein level (Fig. 2B) in SGC7901 cells. These data indicated that psi-CREB1 specifically and significantly inhibited the expression of CREB1 in SGC-7901 cells.

Downregulation of CREB1 inhibits proliferation and colony formation in GC cells. To determine the potential effects of CREB1 on cell proliferation and survival, CCK8 analysis was performed after transfection with psi-CREB1. The results clearly showed that downregulation of CREB1 by psi-CREB1 significantly inhibited cell proliferation in SGC-7901 cells as compared to psi-Ctrl group ( $\mathrm{P}<0.01$, Fig. 3A). Consistent with this result, knockout CREB1 also significantly inhibits colony formation in SGC-7901 cells (Fig. 3B). To reveal the mechanism governing the inhibitory effect of downregulation CREB1 on cell proliferation, cell cycle distribution analysis was performed by flow cytometry. As shown in Fig. 3C, knockout CREB1 lead to an obvious increase in G1/G0 phase and decrease in S phase in SGC-7901 cells compared to psiCtrl group. These results suggested that downregulation of CREB1 inhibited cell proliferation of GC cells by regulating cell cycle distribution.

Downregulation of CREB1 inhibits cell migration and cell invasion of GC cells. To study the effect of CREB1 on metastasis ability of GC cells, migration and invasion assays were performed in SGC-7901 cells transfected with psi-CREB1 or psi-Ctrl by wound healing and invasion chamber assay, respectively. It was found that knockdown of CREB1 could significantly reduce migration (Fig. 4A) and invasion (Fig. 4B) capacity in SGC-7901 cells. 
A

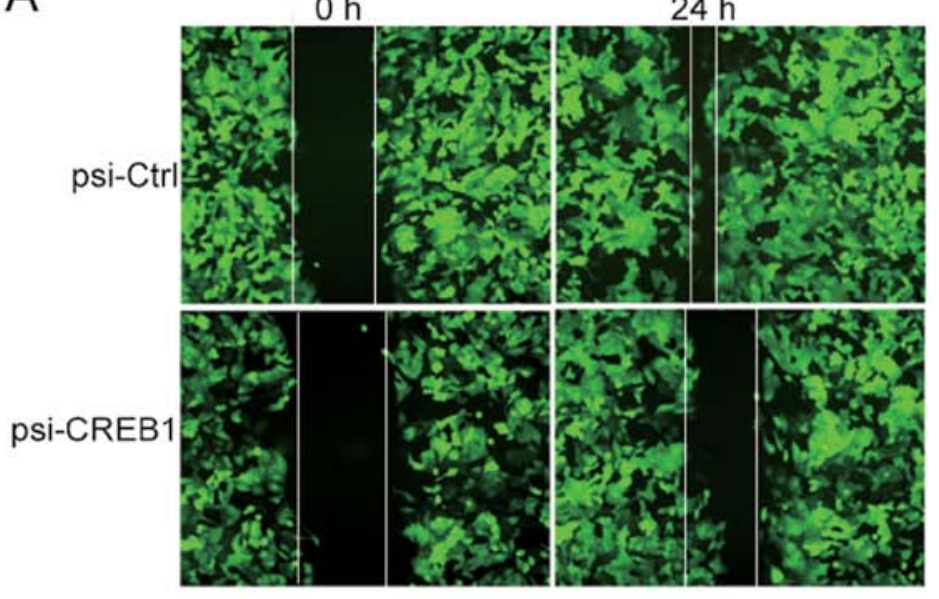

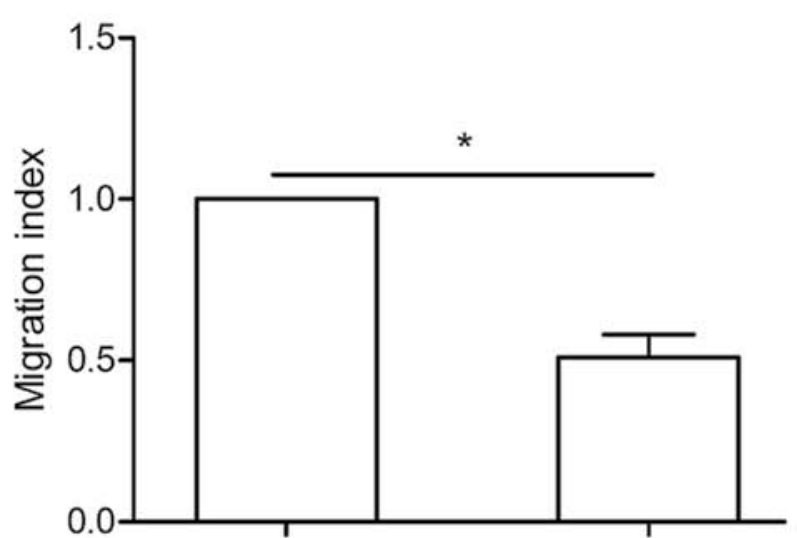

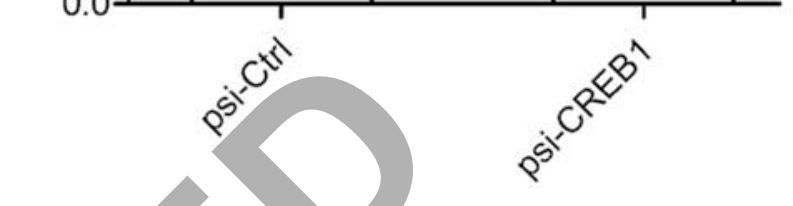

B

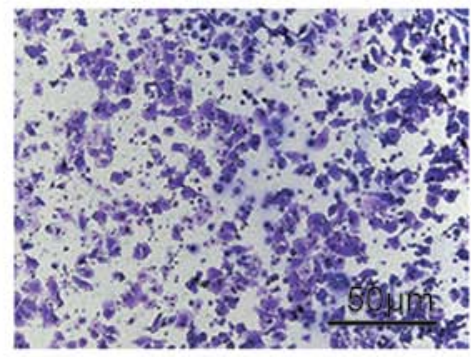

psi-Ctrl
psi-CREB1
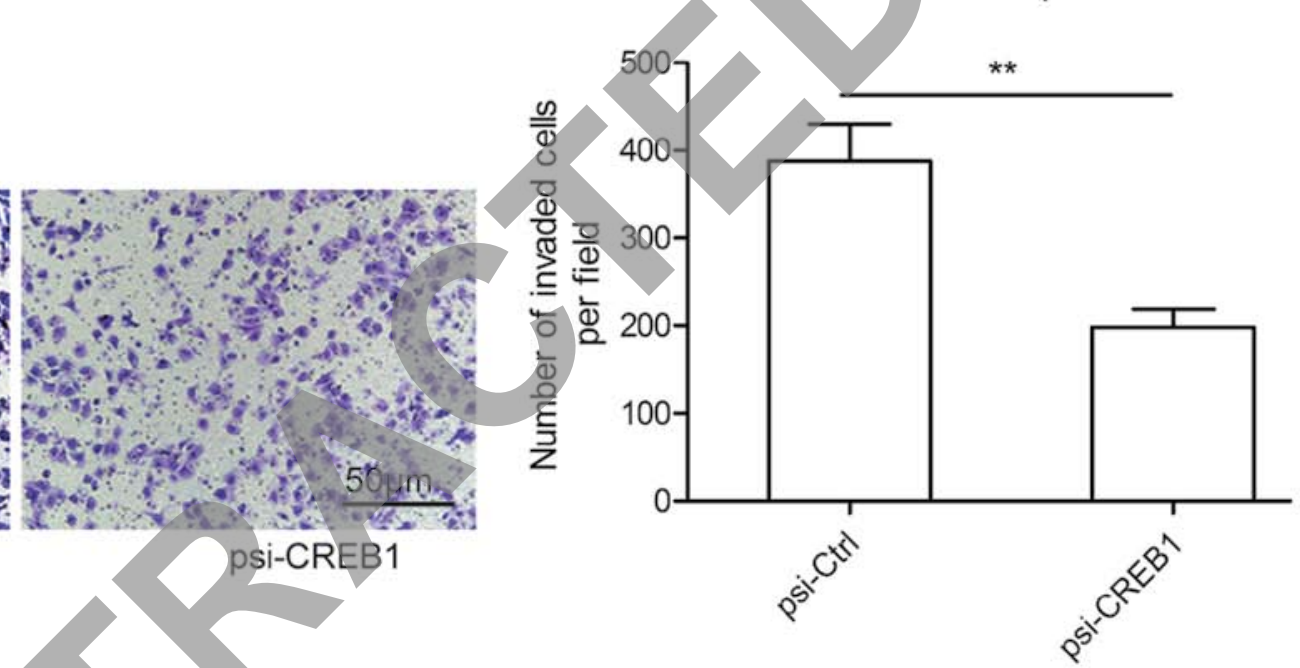

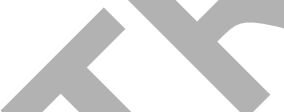

Figure 4. Downregulation of CREB1 inhibits cell migration and invasion of GC cells. (A) Would healing assay was performed to determined cell migration in SGC-7901 cells transfected with psi-CREB1 or psi-Ctrl plasmid. (B) Transwell invasion assay was performed to determined cell invasion in SGC-7901 cells transfected with psi-CREB1 or psi-Ctrl plasmid. ${ }^{*} \mathrm{P}<0.05,{ }^{* *} \mathrm{P}<0.01$.

Downregulation of CREB1 suppresses tumor growth in vivo. We next investigated the effects of downregulation of CREB1 on tumor growth in male BALB mice bearing SGC-7901 tumors cell. It was found that the tumor growth is slower in psi-CREB1 treatment group than that of psi-Ctrl treatment group (Fig. 5A). Tumor growth was monitored for five weeks. On day 35, mice were sacrificed, and then tumor tissues were excised and weighed. We found that the size and weight were markedly reduced in psi-CREB1 treatment group compared with psi-Ctrl treatment group (Fig. 5B and C). Furthermore, we also determined CREB1 expression in tumor tissue by western blotting. We found that CREB1 protein expression obviously decreased in psi-CREB1 treatment group compared with psi-Ctrl treatment group (Fig. 5D). These results suggest that downregulation of CREB1 suppresses tumor growth in vivo.

Downregulation of CREBI inhibited downstream gene of $C R E B 1$ expression in vitro and in vivo. To clarify the molecular mechanisms involved due to downregulation of CREB1 inhibition the growth of human GC in vitro and in vivo, we focused on the effects of knockout CREB1 on its target gene (Bcl-2, cyclin D1, MMP9), which participate in cell apoptosis, cycle and invasion. We measured cyclin D1, Bcl-2 and MMP9 protein expression in SGC7901 cells transfected with psiCREB1 or psi-Ctrl. We found that Bcl-2, cyclin D1, MMP9 protein markedly reduced in SGC7901 cells transfected with psi-CREB1 compared with cells transfected with psi-Ctrl (Fig. 6). In addition, we also detected expression of these proteins in tumor tissues. Consistent with above result, the result demonstrated that cyclin D1, Bcl-2 and MMP9 protein expression were decreased in tumor tissues from psi-CREB1 treatment group compared to psi-Ctrl treatment group (Fig. 6). These results indicate that reduction of CREB1 by siRNA inhibits GC proliferation and invasion, to some extent, by suppressing the target gene of CREB1.

\section{Discussion}

It is well known that the development and progression of human gastric cancer involves multiple genetic changes $(18,19)$. Therefore, identification involving biological molecules for GC 
A

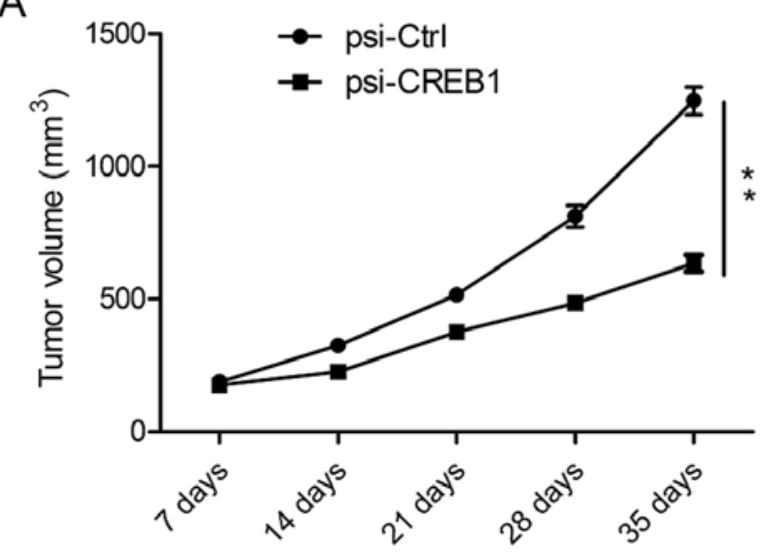

C

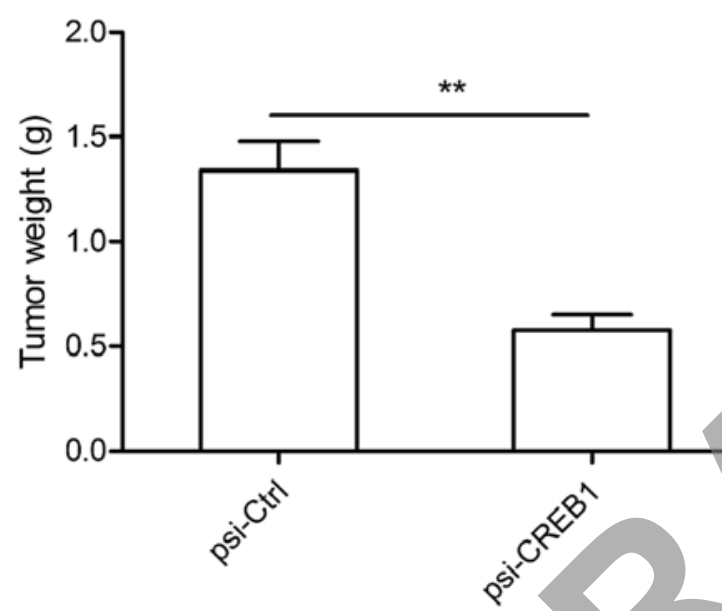

B

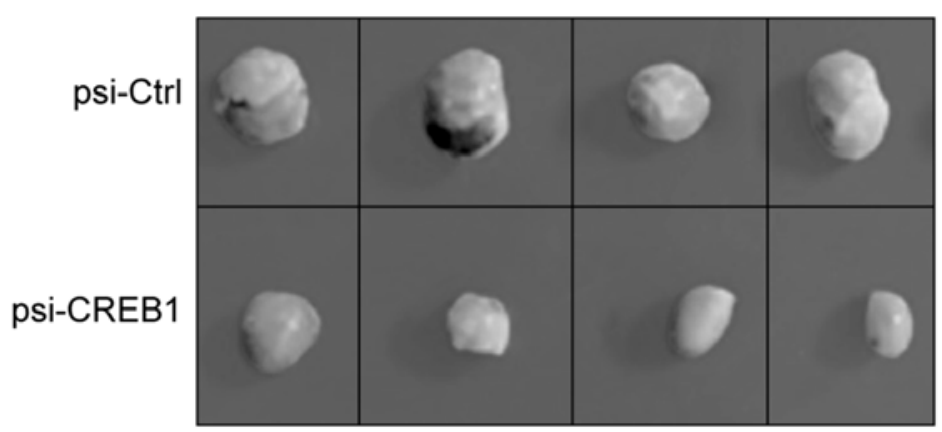

Figure 5. Downregulation of CREB1 suppresses tumor growth in vivo. (A) Growth curves for tumor volumes in xenografts of nude mice were established. (B) Representative graphs of tumor tissues from different treatment groups. (C) Tumor weights from different treatment groups. (D) CREB1 protein expression was determined in tumor tissue by western blotting. GAPDH was used as an internal control. ${ }^{* *} \mathrm{P}<0.01$.
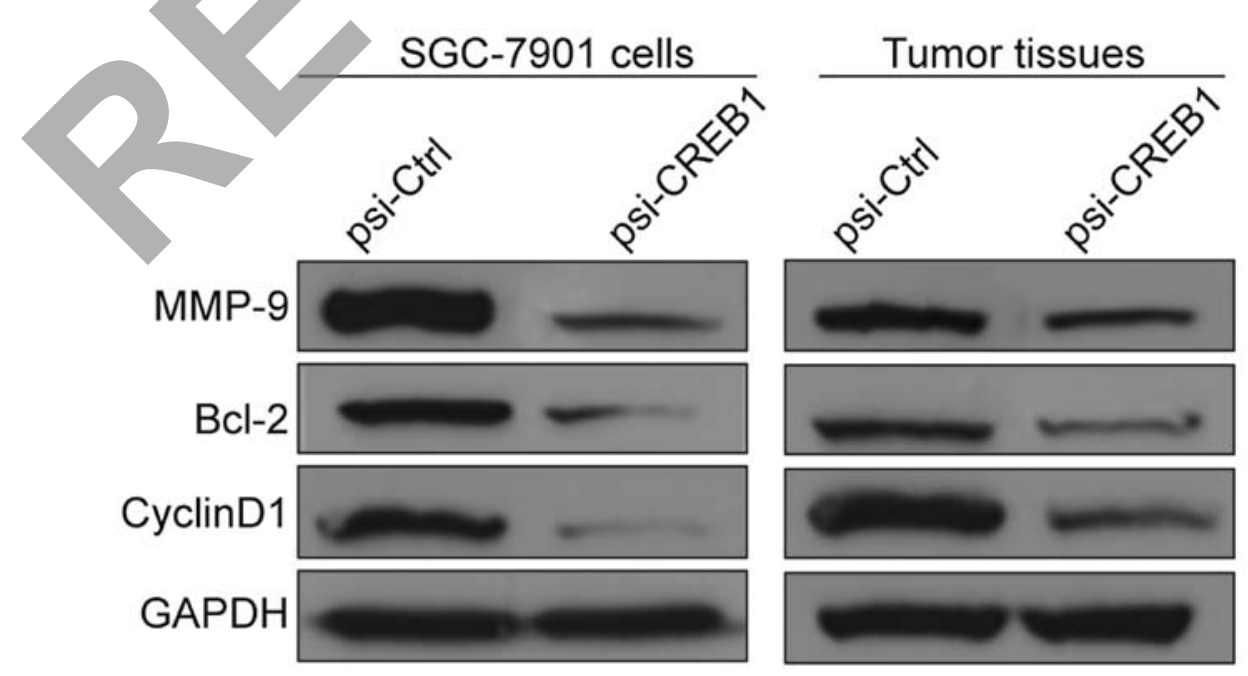

Figure 6. Downregulation of CREB1 inhibits downstream gene of CREB1 expression in vitro and in vivo. Cyclin D1, Bcl-2 and MMP9 protein expression were determined in SGC7901 cells transfected with psi-CREB1 or psi-Ctrl, and in tumor tissues from psi-CREB1 or psi-Ctrl treatment groups. GAPDH was used as an internal control.

progression and development are required to improve existing treatment outcomes in future. Several therapy agents targeting human epidermal growth factor receptor 2 (HER2), epidermal growth factor receptor (EGFR), and STAT3 have been widely 
developed (20-22). In the present study, we found that CREB1 expression was upregulated in gastric cancer lines, and that downregulation of CREB1 expression using RNA silencing approach in SGC-7901 cells significantly suppressed the cell proliferation, colony formation, migration and invasion in vitro, and suppressed tumor growth in vivo. These results implied that CREB1 might be a potential target for the treatment of gastric cancer in the future.

CREB1, located on human chromosome 2q34 (4), has been shown to be upregulated and function as an oncogene in many types of cancer (8-12). For example, Peng et al reported that CREB1 expression was upregulated in glioma tissues, and ectopic expression of CREB1 attenuated the growth suppressive phenotypes of glioma cells caused by overexpression of miR-200 (23). Li et al found that knockdown of CREB1 inhibited colorectal cancer cell proliferation, migration and invasion in vitro (24). It was also shown that small interfering CREB1 inhibited migration of malignant mesothelioma, increased apoptosis and decreased BCL2 and BCL-xL expression (25).

For gastric cancer, previous studies show that CREB1 expression was upregulated and correlated with lymph node metastasis, distant metastasis and tumor stage and poor outcome in gastric cancer (16), and that ectopic expression of CREB1 could overcame the suppressive phenotypes of gastric cancer cells caused by miR-182 (26). However, the detail biological roles of CREB1 in GC remains unknown. The present study is the first to measure expression in four cell lines and find high levels of expression in GC cells. To assess CREB1 function in GC, we constructed the psi-CREB1 vector expressing small hairpin siRNA oligonucleotides targeting CREB1, which efficiently silenced CREB1 in the SGC-7901 cell line. We also found that knockout of CREB1 in GC cells could significantly inhibit cell proliferation, colony formation, migration and invasion in vitro, as well as suppressed tumor growth in vivo. In summary, these results suggest that downregulation of CREB1 inhibits SGC7901 cell growth in vitro and in vivo. Further study is ongoing to validate the biological role of CREB1 in other GC cell lines.

CREB1, a transcription factor containing a 43-kDa basic/ leucine zipper structure, has been shown to play a crucial role in regulating the expression of multiple target genes (27), such as Bcl-2, MMP-9, cyclin D1 and cyclin D3, which are involved in cell cycle, apoptosis, and invasion, thereby contributing to regulating cancer progression. In this study, we investigated whether CREB1 could regulate these genes in GC. We assessed cyclin D1, Bcl-2 and MMP9 protein expression in SGC7901 cells transfected with psi-CREB1 or psi-Ctrl and in tumor tissues from psi-CREB1 or psi-Ctrl treatment groups by western blotting. Our results clearly showed that downregulation of CREB1 significantly inhibited the protein expression in vitro and in vivo, suggesting that knockout CREB1 inhibits GC proliferation and invasion, to some extent, by suppressing the target gene of CREB1.

In conclusion, the present study demonstrated that CREB1 expression was elevated in gastric cancer cell lines and that knockdown of CREB1 in GC cells could significantly inhibit cell proliferation, colony formation, migration and invasion in vitro, as well as suppressed tumor growth in vivo. In addition, knockdown of CREB1 decreased its target genes (cyclin D1, Bcl-2 and MMP-9) expression in vitro and in vivo. Taken together, these results suggest that RNAi-directed targeting of CREB1 may be a beneficial strategy for gastric cancer therapy.

\section{References}

1. Kamangar F, Dores GM and Anderson WF: Patterns of cancer incidence, mortality, and prevalence across five continents: Defining priorities to reduce cancer disparities in different geographic regions of the world. J Clin Oncol 24: 2137-2150, 2006.

2. Torre LA, Bray F, Siegel RL, Ferlay J, Lortet-Tieulent J and Jemal A: Global cancer statistics, 2012. CA Cancer J Clin 65: 87-108, 2015

3. Orditura M, Galizia G, Sforza V, Gambardella V, Fabozzi A, Laterza MM, Andreozzi F, Ventriglia J, Savastano B, Mabilia A, et al: Treatment of gastric cancer. World J Gastroenterol 20: 1635-1649, 2014.

4. Shaywitz AJ and Greenberg ME: CREB: A stimulus-induced transcription factor activated by a diverse array of extracellular signals. Annu Rev Biochem 68: 821-861, 1999.

5. Rudolph D, Tafuri A, Gass P, Hämmerling GJ, Arnold B and Schütź G: Impaired fetal $T$ cell development and perinatal lethality in mice lacking the cAMP response element binding protein. Proc Natl Acad Sci USA 95: 4481-4486, 1998.

6. Mayr B and Montminy M: Transcriptional regulation by the phosphorylation-dependent factor CREB. Nat Rev Mol Cell Biol 2: 599-609, 2001.

7. Sakamoto KM and Frank DA: CREB in the pathophysiology of cancer: implications for targeting transcription factors for cancer therapy. Clin Cancer Res 15: 2583-2587, 2009.

8. Park JK, Park SH, So K, Bae IH, Yoo YD and Um HD: ICAM-3 enhances the migratory and invasive potential of human non-small cell lung cancer cells by inducing MMP-2 and MMP-9 via Akt and CREB. Int J Oncol 36: 181-192, 2010.

9. Zhang M, Xu JJ, Zhou RL and Zhang QY: cAMP responsive element binding protein-1 is a transcription factor of lysosomalassociated protein transmembrane- 4 beta in human breast cancer cells. PLoS One 8: e57520, 2013.

10. Cheng JC, Esparza S, Sandoval S, Shankar D, Fu C and Sakamoto KM: Potential role of CREB as a prognostic marker in acute myeloid leukemia. Future Oncol 3: 475-480, 2007.

11. Guo YH, Wang LQ, Li B, Xu H, Yang JH, Zheng LS, Yu P, Zhou AD, Zhang Y, Xie SJ, et al: Wnt $/ \beta$-catenin pathway transactivates microRNA-150 that promotes EMT of colorectal cancer cells by suppressing CREB signaling. Oncotarget 7: 42513-42526, 2016.

12. Tan X, Wang S, Zhu L, Wu C, Yin B, Zhao J, Yuan J, Qiang B and Peng X: cAMP response element-binding protein promotes gliomagenesis by modulating the expression of oncogenic microRNA-23a. Proc Natl Acad Sci USA 109: 15805-15810, 2012.

13. Singh R, Shankar BS and Sainis KB: TGF- $\beta 1-R O S-A T M-C R E B$ signaling axis in macrophage mediated migration of human breast cancer MCF7 cells. Cell Signal 26: 1604-1615, 2014.

14. Kinjo K, Sandoval S, Sakamoto KM and Shankar DB: The role of CREB as a proto-oncogene in hematopoiesis. Cell Cycle 4: 1134-1135, 2005

15. Wang YW, Chen X, Ma R and Gao P: Understanding the CREB1-miRNA feedback loop in human malignancies. Tumour Biol 37: 8487-8502, 2016.

16. Wang YW, Chen X, Gao JW, Zhang H, Ma RR, Gao ZH and Gao P: High expression of cAMP-responsive element-binding protein 1 (CREB1) is associated with metastasis, tumor stage and poor outcome in gastric cancer. Oncotarget 6: 10646-10657, 2015.

17. Oerlecke I, Bauer E, Dittmer A,Leyh B and Dittmer J: Cyclic AMP enhances TGF $\beta$ responses of breast cancer cells by upregulating TGF $\beta$ receptor I expression. PLoS One 8: e54261, 2013.

18. Mihmanli M, Ilhan E, Idiz UO, Alemdar A and Demir U: Recent developments and innovations in gastric cancer. World $\mathrm{J}$ Gastroenterol 22: 4307-4320, 2016.

19. Digklia A and Wagner AD: Advanced gastric cancer: Current treatment landscape and future perspectives. World J Gastroenterol 22: 2403-2414, 2016. 
20. Cetin B, Gumusay O, Cengiz $\mathrm{M}$ and Ozet A: Advances of molecular targeted therapy in gastric cancer. J Gastrointest Cancer 47: 125-134, 2016.

21. Morishita A, Gong J and Masaki T: Targeting receptor tyrosine kinases in gastric cancer. World J Gastroenterol 20: 4536-4545, 2014.

22. Giraud AS, Menheniott TR and Judd LM: Targeting STAT3 in gastric cancer. Expert Opin Ther Targets 16: 889-901, 2012.

23. Peng B, Hu S, Jun Q, Luo D, Zhang X, Zhao H and Li D: MicroRNA-200b targets CREB1 and suppresses cell growth in human malignant glioma. Mol Cell Biochem 379: 51-58, 2013.

24. Li P, Xue WJ, Feng Y and Mao QS: MicroRNA-205 functions as a tumor suppressor in colorectal cancer by targeting cAMP responsive element binding protein 1 (CREB1). Am J Transl Res 7: 2053-2059, 2015.
25. Shukla A, Bosenberg MW, MacPherson MB, Butnor KJ Heintz NH, Pass HI, Carbone M, Testa JR and Mossman BT: Activated cAMP response element binding protein is overexpressed in human mesotheliomas and inhibits apoptosis. Am J Pathol 175: 2197-2206, 2009.

26. Kong WQ, Bai R, Liu T, Cai CL, Liu M, Li X and Tang H: MicroRNA-182 targets cAMP-responsive element-binding protein 1 and suppresses cell growth in human gastric adenocarcinoma. FEBS J 279: 1252-1260, 2012.

27. Zhang X, Odom DT, Koo SH, Conkright MD, Canettieri G, Best J, Chen H, Jenner R, Herbolsheimer E, Jacobsen E, et al: Genome-wide analysis of cAMP-response element binding protein occupancy, phosphorylation, and target gene activation in human tissues. Proc Natl Acad Sci USA 102: 4459-4464, 2005. 